\title{
Evidências de validade da Escala de Conduta Social para Adolescentes
}

\author{
Caroline Tozzi Reppold ${ }^{1}$, Léia Gonçalves Gurgel \\ Universidade de Ciências da Saúde de Porto Alegre, Porto Alegre-RS, Brasil \\ Claudio Simon Hutz \\ Universidade Federal do Rio Grande do Sul, Porto Alegre-RS, Brasil
}

\section{RESUMO}

No Brasil, são escassos instrumentos para avaliação de conduta social entre adolescentes. Por essa razão, objetivou construir e buscar evidências de validade da Escala de Conduta Social para Adolescentes. Elaborou-se o instrumento baseado em critérios diagnósticos e entrevistas com especialistas e adolescentes. Após isso, buscou-se evidências de validade da estrutura interna e convergente. A amostra constituiu-se por 1093 participantes, 54\% do sexo feminino. Foram usadas a Escala Fatorial de Ajustamento Emocional/Neuroticismo e o Inventário de Habilidades Sociais para a busca de evidências de validade convergente. Como resultado, três fatores foram extraídos: Comportamentos antissociais, Comportamentos pró-sociais, e de Desafio-oposicionismo (com variância explicada, respectivamente, de $14,82 \% ; 5,38 \%$ e $2,81 \%$ ). Diferenças nos três fatores foram encontradas em relação ao sexo e tipo de escola. Houve interação significativa entre idade e sexo, e correlação positiva entre fatores "Comportamento antissocial" e "Desafio-oposicionismo". Concluise que a ECSA apresenta evidências de validade, possibilitando seu uso.

Palavras-chave: adolescência, ajustamento social, comportamento social, avaliação psicológica.

\section{ABSTRACT - Validity evidence of the Scale of Social Conduct for Adolescents}

In Brazil, there are few tools to assess social behavior among adolescents. Therefore, this study aimed to build and seek validity evidence of Social Conduct Scale for Adolescents. We developed the instrument based on diagnostic criteria and interviews with experts and adolescents. Then, we looked for evidence of convergent validity and internal structure. The sample consisted of 1,093 participants, of which 54\% were female. The Factorial Scale of Emotional Adjustment/Neuroticism and Social Skills Inventory were used to the search for evidence of convergent validity. As a result, three factors were extracted: "antisocial behavior", "prosocial behavior", and "challenge-oppositionalism" (with explained variances of $14.82 \%, 5.38 \%$ and $2.81 \%$ respectively). Differences in three factors were found in relation to gender and type of schooling. There was a significant interaction between age and gender, and positive correlation between the factors "antisocial behavior" and "challenge-oppositionalism". We conclude that the ESCA is valid and appropriate for use. Keywords: adolescence; social adjustment; social behavior; psychological assessment.

\section{RESUMEN - La evidencia de la validez de la Escala de Conducta Social para los Adolescentes}

En Brasil, son escasas las herramientas para evaluar el comportamiento social entre los adolescentes. Por lo tanto, este estudio tuvo como objetivo construir y buscar evidencias de validez para esta Escala de Conducta Social de los adolescentes. Se elaboró el instrumento basado en criterios, diagnósticos y entrevistas con especialistas y adolescentes. Se buscaron también evidencias de validez de la estructura interna y convergente. La muestra está formada por 1.093 participantes, 54\% de sexo femenino. Fueron utilizados la Escala Emocional Factorial de Ajuste / Emocional, Inestabilidad Emocional y el Inventario de Habilidades Sociales para la búsqueda de evidencias de validez convergente. Como resultado, se extrajeron tres factores: comportamiento antisocial, conducta pro-social, y desafío-opositor (con variabilidad explicada, respectivamente, $14,82 \%, 5,38 \%$ y $2,81 \%$ ). Las diferencias en los tres factores fueron encontradas con relación al sexo y tipo de escuela. Hubo una interacción significativa entre la edad y el sexo, y correlación positiva entre los factores de "Conducta Antisocial" y "Desafío-opositor". Se llegó a la conclusión de que la Escala de Conducta Social para Adolescentes presenta evidencias de validez, siendo posible su uso.

Palabras clave: adolescencia; ajuste social; comportamiento social; evaluación psicológica.

Os registros contemporâneos sobre violência têm despertado um forte interesse das Ciências da Saúde nas investigações sobre a trajetória de problemas de conduta apresentados por crianças e adolescentes, sobretudo, pela tentativa de prevenir comportamentos antissociais. Projeções estatísticas no campo da saúde coletiva sugerem que os problemas de conduta constituem uma das principais questões a serem enfrentadas pelos países ocidentais 
ao longo dessa década (Marques, 2014; Martins, Silva, Filho, Ribeiro, \& Queiroz, 2013). Esses fenômenos são especialmente preocupantes em razão de sua tendência a persistir no tempo, escalar em gravidade e aumentar a vulnerabilidade dos indivíduos causando interferência no desenvolvimento psicossocial e educacional, gerando consequências na vida adulta (Feitosa, Ricou, Rego, \& Nunes, 2011). Atualmente, é assumida uma perspectiva desenvolvimental para o tema, que evidencia que indicadores prévios de problemas de comportamentos são fortes preditores de violência, dificuldades de interação social, envolvimento criminal, abuso de drogas na vida adulta e outros transtornos psiquiátricos, como a depressão (Silva, 2011).

Em termos gerais, os indicadores de problemas de conduta referem-se a um conjunto de comportamentos relacionado à agressividade, ao oposicionismo, ao negativismo, Á teimosia, à provocação, ao desafio à autoridade, à impulsividade, à humilhação, ao vandalismo e à violação dos direitos alheios. Essas manifestações são pertinentes à investigação diagnóstica de transtornos mentais diversos, como o transtorno da conduta, o transtorno desafiador opositivo e o transtorno de personalidade antissocial (APA, 2002). Estes devem ser analisados longitudinalmente, não apenas em razão da natureza de seus sintomas, como também pela trajetória de prejuízos que a progressão de condutas antissociais aparentemente aceitáveis em suas primeiras manifestações implica (Henriques, 2014).

Estudos sobre comportamentos antissociais apontam que as crianças que, desde cedo, apresentam um padrão de comportamento impulsivo, impaciente, inconsolável, desobediente e socialmente indesejado tendem a apresentar maior risco de desenvolver o transtorno desafiador opositivo (TDO) (Bueno, Santos, \& Moura, 2010). Ou seja, um quadro psicopatológico caracterizado pela dificuldade do sujeito em reconhecer seus próprios erros e por um padrão persistente de ações negativistas, hostis, vingativas e desafiadoras dirigidas a uma figura de autoridade - geralmente um dos pais. No entanto, o curso dos problemas de comportamento não remete necessariamente a uma trajetória crônica, progressiva e psicopatológica (APA, 2002).

Conceitualmente, há um uso indiscriminado das expressões "comportamento antissocial" e "problema da conduta" como sinônimos de comportamento agressivo, delinquência, transtorno de conduta ou problema de externalização. Em comum, esses termos representam comportamentos que implicam incômodo no contexto familiar, escolar e/ou social e que compartilham uma natureza que parece convergir em diversos aspectos ambientais e neurofisiológicos. No entanto, tratam-se de manifestações comportamentais bastante distintas em relação ao seu caráter funcional, à gravidade de suas consequências e ao prognóstico que sugerem (Pacheco \& Hutz, 2009).
Os transtornos externalizantes abrangem diagnósticos de transtornos mentais nos quais estão presentes comportamentos agressivos, impulsivos ou delinquentes. Já o comportamento antissocial não necessariamente envolve um diagnóstico psiquiátrico ou um ato ilegal, embora se caracterize por atos que envolvam agressividade auto ou heterodirigida, conduta desafiadora, ou outras características que impliquem conflitos com o meio e, por consequência, prejuízo à adaptação social. Ele pode ser definido como um padrão de resposta que objetiva gratificações imediatas e a neutralização das exigências do ambiente social, diretamente relacionados à ação de uma outra pessoa (Pacheco, Alvarenga, Reppold, Piccinini, \& Hutz, 2005).

A agressão, nesse sentido, é uma expressão física ou verbal que gera prejuízos físicos ou psicológicos aos sujeitos (Paula, Kodato, \& Dias, 2013). A agressividade deve ser diferenciada da violência em função do impacto negativo que as condutas violentas trazem às suas vítimas. Nesse sentido, a violência é considerada como um comportamento exclusivamente humano, melhor definido como uma característica própria de algumas formas de agressão que causam, deliberadamente, graves danos a outro indivíduo. Esses danos podem envolver opressão, ameaça, constrangimento físico ou moral ou qualquer ação exercida contra a vontade alheia (premeditada ou não) (Krintensen, Lima, Ferlin, Flores, \& Hackmann, 2003).

Os dados epidemiológicos brasileiros revelam um aumento da incidência de casos de agressividade juvenil e de violência doméstica, a partir dos anos 70. Esses índices referem-se a situações de hostilidade familiar e escolar, brigas violentas entre pares, sérias violações de regras e outras expressões de comportamentos impulsivos e antissociais mais graves, como o cometimento de atos delinquentes ou homicidas por adolescentes (de Deus, 2013; Macedo, Paim, Silva, \& Costa, 2001), além de uso de álcool e drogas (Almeida et al., 2014). Nessa linha, pode-se observar que a influência do contexto social sobre o desenvolvimento de problemas de conduta pode produzir seus efeitos desde os primeiros anos de vida da criança. Estudos apontam que as crianças pertencentes a famílias com baixo nível socioeconômico tendem a apresentar maior prevalência de problemas de conduta precoce (Borsa, Souza, \& Bandeira, 2011). Ainda, a falta de monitoramento propicia a relação com pares desviante $\mathrm{e}$ a incidência de atos de bullying e delinquência e, consequentemente, uma maior rejeição social e baixo desempenho escolar (Silva et al., 2012). Laird, Pettit, Dodge, e Bates (2005), em um estudo longitudinal, indicam que, em diferentes culturas, adolescentes descritos por si ou por seus pais como envolvidos com amigos desviantes aos 13-16 anos tinham, significativamente, mais chances de apresentar comportamentos delinquentes aos 17-18 anos, bem como menores índices de cooperação e aceitação social junto a outros adolescentes. Os 
estudos realizados sobre ajustamento juvenil feminino, por exemplo, também enfatizam que o número de meninas que apresentam problemas de externalização (como TDAH e transtornos disruptivos) é bastante reduzido em comparação aos meninos (Pedrini \& Frizzo, 2010).

Em relação aos aspectos neuropsicológicos relacionados ao tema, o estudo de Teichner e Golden (2000) mostra que, de forma recorrente, a literatura indica a associação existente entre o transtorno de conduta e prejuízos relacionados a habilidades verbais. Observa-se também prejuízos relacionados a diferentes funções executivas (atenção, flexibilidade cognitiva, formação de objetivos, julgamento, abstração, planejamento da sequência de comportamentos motores, inibição de comportamentos impulsivos ou inadequados e automonitoramento). Com bases nesses prejuízos, é possível distinguir quatro subgrupos que melhor explicam a heterogeneidade dos danos neuropsicológicos associados aos problemas de conduta na adolescência. Os quatros grupos são formados, respectivamente, por adolescentes com lesões/ disfunções nas áreas subcorticais, no hemisfério dominante parieto-temporal, no hemisfério não dominante parieto-temporal e nas regiões pré-frontais (Teichner \& Golden, 2000).

Os comportamentos pró-sociais também devem ser considerados nesse contexto, estando relacionados com as ações que promovem o bem-estar dos sujeitos. Esse tipo de comportamento gera benefícios ao desenvolvimento das crianças e adolescentes, fornecendo apoio social, apoio emocional e proteção. Nesse caso, avaliações adequadas do comportamento e conduta de crianças e adolescentes devem considerar também as ações assertivas pró-sociais e são poucos os estudos voltados para o tema (Costa \& Cavalcante, 2012; Del Prette \& Del Prette, 2008).

Desse modo, o diagnóstico de problemas de conduta depende, também, em grande parte, de observações clínicas e entrevistas trianguladas. Todavia, a consideração dos dados relativos à percepção dos adolescentes sobre comportamentos agressivos, dissimulados ou delinquentes - os quais nem sempre são comportamentos evidentes e conhecidos por terceiros - é um elemento clínico importante para avaliação da gravidade de possíveis padrões de comportamento antissociais que se desviam dos padrões do desenvolvimento típico, além das características da família e dos contextos relacionados (Nardi \& Dell'Aglio, 2012; Souza \& Resende, 2012). No campo internacional, alguns dos instrumentos comumente utilizados para avaliação de problemas de conduta entre adolescentes são o YSR - Inventário de Autoavaliação para Adolescentes de 11 a 18 anos (adaptado para a população brasileira por Rocha, 2012), a Conners Conduct Problem Scale, as Rutter Scales (versões A2 para pais e B2 para professores), a Disruptive Behavior Disorder Scale, o Child Behavior Questionnaire - CBQ e o Millon Adolescent Clinical Inventory, dentre outros. Já no
Brasil, não são encontrados instrumentos aprovados pelo Conselho Federal de Psicologia para uso clínico. Assim, a avaliação dessa medida baseou-se na comparação de resultados obtidos em instrumentos divergentes, tais como o Inventário de Habilidades Sociais, validado por Del Prette e Del Prette (2003).

Frente à escassez de testes validados disponíveis para uso clínico dos profissionais da área da saúde, sobretudo no caso dos psicólogos, compreende-se que a formulação de uma escala de autorrelato relativa a problemas de condutas pode ser de grande valia para investigação diagnóstica e para o campo científico. Esse material possibilita recursos para triangulação dos dados e análise do julgamento crítico dos adolescentes envolvidos em persistentes comportamentos antissociais. Objetivou-se, portanto, com este estudo, apresentar a construção e busca de evidências de validade da Escala de Conduta Social para Adolescentes.

\section{Método}

\section{Participantes}

A amostra total foi constituída por 1093 participantes (idade média $=14,4 ; D P=1,72$ ), sendo $54 \%$ do sexo feminino. Os participantes compuseram uma amostra de conveniência. Todos os sujeitos estavam matriculados em dezessete escolas públicas e privadas de Porto Alegre e região metropolitana, região sul do Brasil, em séries compatíveis com a idade. O número de participantes foi definido em função do número de itens que compunham o instrumento original. O cálculo do tamanho amostral seguiu o critério da razão itens/participantes, usualmente utilizado quando são necessárias análises fatoriais (Pasquali, 1999).

\section{Instrumentos}

Escala de Conduta Social para Adolescentes. Para a elaboração desse instrumento, inicialmente, foram consideradas as diretrizes diagnósticas no Manual Diagnóstico de Transtornos Mentais (APA, 2002), diretrizes da International Test Comission (ITC, 2000) e estudos empíricos acerca da saúde psicológica conduzidos sob a abordagem da Epidemiologia Clínica, tais como APA (2002), Pacheco \& Hutz (2009), Pacheco et al. (2005) e Laird et al. (2005). Para estabelecer validade de conteúdo, foram realizadas entrevistas com oito profissionais da área da saúde juvenil sobre a manifestação clínica do construto, como psicólogos e psiquiatras especialistas. Além disso, foram realizadas entrevistas com 24 adolescentes entre 12 e 17 anos para coletar sugestões sobre os modos mais adequados e inteligíveis à população juvenil de expressar, em forma de itens, comportamentos representativos do construto. A validade de conteúdo do teste foi evidenciada em diferentes estudos de validade semântica, validade aparente e avaliação por juízes. A escala em questão busca avaliar comportamentos pró-sociais e antissociais, 
de modo a apresentar uma avaliação dos problemas de conduta dos sujeitos.

Nesse contexto, a escala elaborada no presente estudo apresenta itens, como: "Gosto de assistir a cenas de violência", "Gosto de implicar com meus amigos", "Tenho comportamentos considerados violentos", "Já bati no meu pai ou na minha mãe", "Não vejo problemas em mentir para conseguir o que quero", "Acho que as pessoas, em geral, têm medo de mim", "Geralmente me sinto culpado depois de ter feito algo errado", "Consigo controlar minha raiva", "Cumpro as combinações que faço", "Gosto de ter a última palavra em uma discussão", "Sou teimoso", "Adoro desafiar as regras" e "Frequentemente pareço estar de mal com o mundo". O instrumento foi organizado em três partes. A primeira conta com instruções para o preenchimento da escala; a segunda conta com os itens propriamente ditos que compõem cada teste; e a terceira refere-se à folha de resposta, com dados demográficos do participante, incluindo a idade e a escolaridade, e uma escala Likert para as respostas, em que 1 significaria discordo completamente/não tem nada a ver comigo e 5 significaria concordo completamente/tem tudo a ver comigo.

A Escala Fatorial de Ajustamento Emocional/ Neuroticismo (EFN; Hutz \& Nunes, 2001) é indicada para indivíduos acima de 17 anos de idade. Possui 82 itens e é respondida por meio de uma escala Likert de sete pontos. Baseada no modelo dos Cinco Grandes Fatores da personalidade, a escala avalia o construto Estabilidade emocional a partir de quatro dimensões: Vulnerabilidade, Desajustamento psicossocial, Ansiedade e Depressão. A escala apresenta propriedades psicométricas adequadas, sendo que a subescala Vulnerabilidade apresenta componente que explica $32,46 \%$ da variância total (eigenvalue =7,46), com índice KMO de 0,91 e teste de esfericidade de Bartlett com resultado significativo $(p<0,001)$. A carga componencial dos itens está entre 0,34 e 0,70 (Zanon \& Hutz, 2009).

O Inventário de Habilidades Sociais (IHS; Del Prette \& Del Prette, 2003) é um instrumento de autorrelato, indicado para adultos e adolescentes acima de 17 anos de idade, com formação mínima de ensino médio. Avalia o repertório de comportamentos sociais usualmente requeridas em situações interpessoais cotidianas para um desempenho competente. É constituído por 38 itens, respondidos por meio de uma escala Likert de cinco pontos. Apresenta índices de validade, fidedignidade e consistência interna satisfatórios, sendo adequado psicometricamente (Carneiro, Falcone, Clark, Del Prette, \& Del Prette, 2007). Ambos os instrumentos mencionados foram utilizados na busca de evidências de validade convergente da Escala de Conduta Social para Adolescentes.

\section{Procedimentos}

A coleta dos dados foi realizada em sala de aula, em grupos de, no máximo, 40 alunos. $\mathrm{O}$ ambiente era silente e livre de interferências externas. A administração do instrumento incluía uma breve explicação sobre os objetivos do estudo, a leitura conjunta das instruções e eventuais explicações adicionais solicitadas. Após essa etapa, o participante respondia à escala de maneira individual. Os outros dois instrumentos eram aplicados no mesmo momento e a aplicação foi realizada de forma espiralada, a fim de evitar viés de aplicação.

Previamente foi solicitada a emissão do Termo de Consentimento Livre e Esclarecido do responsável legal pelo participante. Os adolescentes eram informados, antes do início da testagem, que sua participação era voluntária, que suas respostas seriam anônimas e que eles poderiam interromper sua participação em qualquer momento da coleta. O presente projeto de pesquisa foi registrado no CONEP e aprovado pelo CEP do Hospital de Clínicas de Porto Alegre, sob número 05.502.

\section{Análise de Dados}

Para estabelecer as evidências de validade baseadas na estrutura interna, realizou-se o cálculo dos alfas de Cronbach da escala. Para estabelecer as evidências de validade convergente da Escala de Conduta Social para Adolescentes, foram eleitos testes padrão ouro já validados para a população brasileira e aprovados pelo Conselho Federal de Psicologia: Escala Fatorial de Ajustamento Emocional/Neuroticismo (EFN; Hutz \& Nunes, 2001) e Inventário de Habilidades Sociais (IHS; Del Prette \& Del Prette, 2003). Os dados dos testes foram submetidos à técnica de correlação de Pearson. Do total, 102 adolescentes responderam a EFN (média de idade de 15,9 e desvio padrão de 0,91 ), sendo 54,1 do sexo feminino; e 42 adolescentes responderam ao IHS (idade média de 16,2 anos; desvio padrão de 1,09), sendo $61,7 \%$ do sexo feminino. O tempo aproximado utilizado para responder à escala foi de 15 minutos.

\section{Resultados}

Para a busca de evidências de validade baseadas na estrutura interna, foram realizadas análises exploratórias. Três fatores foram extraídos para a Escala de Conduta Social para Adolescentes. O primeiro fator, Comportamentos antissociais, com 40 itens e um eigenvalue igual a 14,11 , teve uma variância explicada de $14,82 \%$. O segundo, Comportamentos pró-sociais, com 23 itens e um eigenvalue igual a 5,64, explicou $5,38 \%$ da variância; e o terceiro, Desafio-oposicionismo, com 17 itens e um eigenvalue igual a 2,96, respondeu por $2,81 \%$ da variância total. No conjunto, os três fatores somaram 80 itens e explicaram $23,41 \%$ da variância total das respostas. As cargas fatoriais variaram de 0,30 a 0,61 . Ao total, a escala apresentou 80 itens.

A extração de três fatores foi corroborada pela análise teórica dos itens agrupados. Os itens reunidos no 
primeiro fator referem-se a Comportamentos antissociais relacionados aos marcadores do transtorno da conduta. Os do segundo fator referem-se a indicadores de comportamento pró-social. Já as sentenças do terceiro fator representam comportamentos de iniciativa e reação diante de situações que envolvam demanda social. Esses comportamentos podem se qualificar, em situações extremas e persistentes, como indicadores de desafio e oposicionismo.
Nesse sentido, para a escala proposta no presente estudo, não há indicação para a soma de um escore total, nem a necessidade de inversão das respostas para o levantamento das subescalas. Todos os fatores foram compostos por itens de mesma direção. O levantamento de cada fator foi realizado por meio da soma das respostas emitidas nos itens que o compõem. A média, o desvio padrão e a análise de consistência interna dos grupos de itens relacionados com cada fator podem ser verificados na Tabela 1 .

Tabela 1

Resultados Descritivos da Escala de Conduta Social

\begin{tabular}{lccccc}
\hline & $N^{\circ}$ de itens & $M$ & $D P$ & $\alpha$ & Corr. média interitem \\
\hline Fator 1 & 40 & 73,3 & 23,10 & 0,92 & 0,21 \\
Fator 2 & 23 & 85,5 & 13,89 & 0,83 & 0,18 \\
Fator 3 & 17 & 47,3 & 12,63 & 0,84 & 0,24 \\
\hline
\end{tabular}

Testes de comparação de médias foram executados para verificar se o efeito das variáveis sexo, idade, série e tipo de escola produziam diferenças significativas nos escores. Por meio do teste $t$, observou-se que meninos e meninas responderam de forma significativamente diferente aos três fatores (Fator 1: $t=-11,29, g l=987$, $p<0,01$; Fator 2: $t=5,07, g l=984, p<0,01$; Fator 3: $t=-2,83, \mathrm{gl}=1051, p<0,01)$. Diferenças nos três fatores também foram encontradas quando comparados alunos de escola pública e privada (Fator 1: $t=-7,34$, $g l=976, p<0,01$; Fator 2: $t=3,54, g l=977, p<0,01$; Fator 3: $t=-6,96, g l=1043, p<0,01)$.

Em relação à idade, observa-se que os participantes de 12 anos apresentaram um escore significativamente menor do que os de 13,14, 15 e 16 anos de idade nos fatores 1 e 3 . No fator 2, os adolescentes de 13 anos apresentaram um escore significativamente menor que os de 15,16 e 17 anos $(p<0,05)$. Na Tabela 2, pode-se visualizar as médias e desvio padrão dos escores nos três fatores obtidos nas ANOVAs. Como as análises de variância mostraram diferenças, a variável Idade foi agrupada em dois grupos (maiores ou menores de 15 anos) e ANOVAs foram realizadas para cada um dos três fatores. Os resultados mostraram interações significativas (Fator 1: $F_{5,979}=25,97, p<0,01$; Fator $2: F_{5,977}=12,33, p<0,01$; Fator 3: $\left.F_{5,1042}=6,45, p<0,01\right)$ entre idade e sexo.

Tabela 2

Comparação de Médias pelas Variáveis Demográficas da Escala de Conduta Social

\begin{tabular}{|c|c|c|c|c|c|c|c|c|c|}
\hline \multirow{2}{*}{ Idade } & \multicolumn{3}{|c|}{$\begin{array}{c}\text { Fator } 1 \\
\text { Comportamento antissocial }\end{array}$} & \multicolumn{3}{|c|}{$\begin{array}{c}\text { Fator } 2 \\
\text { Comportamento Pró-social }\end{array}$} & \multicolumn{3}{|c|}{$\begin{array}{c}\text { Fator } 3 \\
\text { Desafio-oposicionismo }\end{array}$} \\
\hline & $M$ & $n$ & DP & $M$ & $n$ & $D P$ & $M$ & $n$ & $D P$ \\
\hline \multicolumn{10}{|l|}{ Sexo } \\
\hline Masculino & 81,9 & 452 & 25,77 & 83,2 & 461 & 14,09 & 48,9 & 488 & 12,40 \\
\hline Feminino & 66,1 & 537 & 17,74 & 87,6 & 525 & 13,38 & 46,6 & 565 & 12,77 \\
\hline \multicolumn{10}{|l|}{ Idade } \\
\hline 11 & 69,3 & 29 & 21,37 & 82,2 & 27 & 12,44 & 42,4 & 38 & 13,03 \\
\hline 12 & 62,5 & 66 & 15,90 & 81,1 & 64 & 16,19 & 38,8 & 76 & 12,16 \\
\hline 13 & 78,9 & 66 & 28,30 & 78,4 & 70 & 14,32 & 47,8 & 84 & 12,47 \\
\hline 14 & 74,9 & 131 & 23,66 & 83,4 & 124 & 14,29 & 47,6 & 133 & 14,13 \\
\hline 15 & 74,7 & 241 & 23,05 & 87,1 & 245 & 13,36 & 48,3 & 247 & 11,77 \\
\hline 16 & 73,6 & 282 & 23,01 & 86,8 & 284 & 13,34 & 50,0 & 289 & 12,13 \\
\hline 17 & 71,7 & 173 & 21,49 & 88,1 & 172 & 12,86 & 47,9 & 184 & 11,84 \\
\hline \multicolumn{10}{|l|}{ Série } \\
\hline $5 \mathrm{EF}$ & 69,2 & 31 & 21,08 & 75,3 & 31 & 13,77 & 39,2 & 40 & 11,05 \\
\hline $6 \mathrm{EF}$ & 70,2 & 89 & 22,86 & 80,8 & 88 & 15,92 & 43,9 & 104 & 14,47 \\
\hline $7 \mathrm{EF}$ & 78,8 & 59 & 29,79 & 83,7 & 56 & 14,44 & 45,0 & 63 & 12,27 \\
\hline $8 \mathrm{EF}$ & 77,6 & 85 & 26,51 & 80,2 & 87 & 13,50 & 47,0 & 96 & 13,64 \\
\hline
\end{tabular}


Tabela 2 (continuação)

Comparação de Médias pelas Variáveis Demográficas da Escala de Conduta Social

\begin{tabular}{|c|c|c|c|c|c|c|c|c|c|}
\hline \multirow[t]{2}{*}{ Idade } & \multicolumn{3}{|c|}{$\begin{array}{c}\text { Fator } 1 \\
\text { Comportamento antissocial }\end{array}$} & \multicolumn{3}{|c|}{$\begin{array}{c}\text { Fator } 2 \\
\text { Comportamento Pró-social }\end{array}$} & \multicolumn{3}{|c|}{$\begin{array}{c}\text { Fator } 3 \\
\text { Desafio-oposicionismo }\end{array}$} \\
\hline & $M$ & $n$ & $D P$ & $M$ & $n$ & $\mathrm{DP}$ & $M$ & $n$ & $\mathrm{DP}$ \\
\hline $1 \mathrm{EM}$ & 71,0 & 183 & 22,17 & 86,1 & 186 & 13,73 & 47,3 & 187 & 12,70 \\
\hline $2 \mathrm{EM}$ & 76,2 & 289 & 23,70 & 87,0 & 289 & 13,67 & 49,1 & 299 & 12,02 \\
\hline 3 EM & 70,9 & 247 & 20,03 & 88,4 & 245 & 12,07 & 50,1 & 257 & 11,48 \\
\hline \multicolumn{10}{|l|}{ Escola } \\
\hline Pública & 69,6 & 647 & 21,27 & 86,6 & 657 & 14,22 & 45,8 & 688 & 12,49 \\
\hline Particular & 80,8 & 331 & 25,06 & 83,3 & 322 & 13,15 & 51,4 & 357 & 12,20 \\
\hline \multicolumn{10}{|l|}{ Sexo vs idade } \\
\hline Masc. us $<15$ & 83,2 & 130 & 27,60 & 78,6 & 137 & 14,51 & 46,9 & 154 & 13,03 \\
\hline Masc. us $>15$ & 81,1 & 320 & 24,71 & 85,2 & 322 & 13,49 & 49,8 & 331 & 11,97 \\
\hline Fem. us $<15$ & 63,8 & 162 & 15,56 & 84,4 & 148 & 14,29 & 43,5 & 177 & 13,99 \\
\hline Fem. us $>15$ & 67,2 & 373 & 18,56 & 89,0 & 376 & 12,80 & 48,2 & 386 & 11,94 \\
\hline
\end{tabular}

Nota. EF=Ensino Fundamental; EM=Ensino Médio; $n=$ número de indivíduos; $D P=$ desvio padrão; us - versus

Quanto à distribuição das respostas, análises de dispersão mostraram que os grupos relacionados com cada fator tiveram uma distribuição normal (Liliefords $p<0,01)$. O Fator 1 apresentou assimetria igual a 1,17 e curtose de 1,79. No Fator 2, esses índices foram, respectivamente, iguais a $-0,46$ e 0,19 e, no Fator 3 , respectivamente iguais a 0,04 e $-0,50$.

Para a busca de evidências de validade convergente, as correlações obtidas entre a escala criada e os instrumentos padrão ouro podem ser verificadas na Tabela 3. Na comparação da Escala de Conduta Social com o fator Desajustamento psicossocial da EFN, constatou-se uma forte correlação positiva com fatores Comportamento antissocial e Desafio-oposicionismo, e uma correlação negativa com o fator Comportamento pró-social. Uma correlação negativa foi observada entre o fator Comportamento pró-social e o fator Ansiedade do EFN. O fator Desafio-oposicionismo apresentou também uma correlação positiva com os fatores Ansiedade e Depressão da EFN.

Na comparação da Escala de Conduta Social com o Inventário de Habilidades Sociais, o fator Desafiooposicionismo mostrou uma correlação significativa positiva com os fatores Conversação social e desenvoltura social e Autoexposição a desconhecidos e situações novas. Ainda, uma correlação significativa foi observada entre a subescala Comportamento pró-social e o fator Enfrentamento/ autoafirmação com risco do IHS (Tabela 3).

Tabela 3

Correlação entre a Escala de Conduta Social e os Instrumentos Considerados Padrão Ouro

\begin{tabular}{|c|c|c|c|}
\hline Padrão ouro & Fator 1 & Fator 2 & Fator 3 \\
\hline EFN - Fator Vulnerabilidade Psicológica & $\begin{array}{c}r=-0,04 \\
n=92\end{array}$ & $\begin{array}{c}r=-0,02 \\
n=91\end{array}$ & $\begin{array}{c}r=-0,17 \\
p<0,09 \\
n=96\end{array}$ \\
\hline EFN - Fator Desajustamento psicossocial & $\begin{array}{c}r=0,57^{* *} \\
n=94\end{array}$ & $\begin{array}{c}r=-0,43^{* *} \\
n=92\end{array}$ & $\begin{array}{c}r=0,56^{* *} \\
n=97\end{array}$ \\
\hline EFN - Fator Ansiedade & $\begin{array}{c}r=0,15 \\
n=95\end{array}$ & $\begin{array}{c}r=-0,24^{* *} \\
n=94\end{array}$ & $\begin{array}{c}r=0,43^{* *} \\
n=99\end{array}$ \\
\hline EFN - Fator Depressão & $\begin{array}{l}r=0,09 \\
\mathrm{n}=92\end{array}$ & $\begin{array}{c}r=-0,10 \\
\mathrm{n}=90\end{array}$ & $\begin{array}{c}r=0,23^{*} \\
n=95\end{array}$ \\
\hline $\begin{array}{l}\text { IHS - Fator Enfrentamento/ } \\
\text { Autoafirmação com Risco }\end{array}$ & $\begin{array}{c}r=-0,02 \\
n=32\end{array}$ & $\begin{array}{c}r=0,38^{*} \\
n=32\end{array}$ & $\begin{array}{c}r=0,07 \\
n=34\end{array}$ \\
\hline IHS - Fator Autoafirmação de Afeto Positivo & $\begin{array}{c}r=-0,18 \\
p<0,10 \\
n=33\end{array}$ & $\begin{array}{c}r=0,28 \\
n=33\end{array}$ & $\begin{array}{c}r=0,09 \\
n=34\end{array}$ \\
\hline
\end{tabular}


Tabela 3 (continuação)

Correlação entre a Escala de Conduta Social e os Instrumentos Considerados Padrão Ouro

\begin{tabular}{|c|c|c|c|}
\hline Padrão ouro & Fator 1 & Fator 2 & Fator 3 \\
\hline IHS - Fator Conversação e Desenvoltura Social & $\begin{array}{l}r=0,10 \\
\mathrm{n}=34\end{array}$ & $\begin{array}{l}r=-0,01 \\
\mathrm{n}=34\end{array}$ & $\begin{array}{c}r=0,43^{* *} \\
n=35\end{array}$ \\
\hline $\begin{array}{l}\text { IHS - Fator Autoexposição a } \\
\text { Desconhecidos e Situações Novas }\end{array}$ & $\begin{array}{c}r=0,13 \\
n=33\end{array}$ & $\begin{array}{c}r=-0,01 \\
n=33\end{array}$ & $\begin{array}{c}r=0,37^{*} \\
n=34\end{array}$ \\
\hline IHS - Autocontrole da Agressividade & $\begin{array}{c}r=-0,01 \\
n=34\end{array}$ & $\begin{array}{c}r=0,21 \\
n=34\end{array}$ & $\begin{array}{c}r=-0,06 \\
n=35\end{array}$ \\
\hline
\end{tabular}

Nota. ${ }^{*} p>0,05 ;{ }^{* *} p>0,01$

\section{Discussão}

Os itens que se agruparam no primeiro fator referem-se a condutas agressivas ou humilhantes (bullying), vandalismo, violação de direitos alheios e de normas morais, envolvimento em brigas, intimidação, porte de armas e defraudações. Em geral, caracterizam-se como marcadores do Transtorno da Conduta. Os itens do segundo fator referem-se a Comportamentos pró-sociais, ou seja, ações que envolvem expressão de empatia, solidariedade, atenção às solicitações, às combinações e aos direitos alheios, busca de bem-estar coletivo e controle de afetos negativos. Já os itens do terceiros fator representam comportamentos relacionados à iniciativa e à tentativa de controle da situação, que podem dar origem a condutas inassertivas diante de demandas sociais em algumas situações ou a condutas de desafio, oposicionismo e agressividade (velada ou verbal) em circunstâncias extremas.

Dependendo da frequência e intensidade de ocorrência, os comportamentos descritos nas sentenças podem representar busca de dominância, iniciativa, persuasão, tendência a não se submeter passivamente ao controle externo, desejo de superar desafios, ou ainda ser associados a comportamentos antissociais evidentes e destrutivos. Isso mostra que, numa perspectiva dimensional, um escore médio-alto no fator 3 pode indicar características de personalidade típicas de pessoas que apresentam altos padrões de ambição e empenho e forte desejo de autorrealização, e não necessariamente comportamentos violentos ou antissociais.

Em relação às diferenças de média observadas por sexo, idade, série e tipo de escola, pode-se afirmar que os resultados são coerentes com descrição epidemiológica dos estudos sobre o tema. Verifica-se que os itens nos quais os meninos obtêm maior escore são, principalmente, as sentenças dos fatores 1 e 3 . A expectativa é que as diferenças entre os sexos nesses indicadores sejam mais pronunciadas na infância e diminuam ao longo do desenvolvimento (Koch \& Gross, 2005), como pode ser observado, de fato, no resultado da ANOVA por idade.
A diferença relacionada ao tipo de escola também pode ser justificada. Os resultados mostraram que os participantes alunos de escola pública apresentam maiores escores nos Fatores 1 e 3 e menor escore no Fator 2. Provavelmente isso ocorre porque, com o passar do tempo, os alunos de escola pública que manifestam condutas antissociais e oposicionistas têm maior chance de ser expulso da escola ou espontaneamente evadir para obtenção de recursos materiais que lhe são mais escassos (Netto, Rodrigues, Costa, Santos, \& Tatagiba, 2012). Além disso, é preciso considerar o alto percentual de pais de classe média e média alta que são classificados por seus filhos como negligentes, como no estudo de Benchaya, Bisch. Moreira, Ferigolo, e Barros (2011) com usuários adolescentes de drogas.

As correlações obtidas evidenciam a validade convergente/divergente da escala criada. As correlações significativas ocorreram nos fatores esperados, quando cruzados os resultados com a EFN. Indivíduos que apresentam altos escores nas subescalas Comportamento antissocial e Desafio-oposicionismo tendem a apresentar também altos escores no fator Desajustamento emocional da EFN. Em contraponto, indivíduos com alto escore na escala de comportamento pró-social, tendem a apresentar menos indicadores de desajustamento psicossocial na Escala de Ajustamento Emocional/Neuroticismo. Essa subescala do EFN avalia indicadores de personalidade relacionados ao Transtorno de personalidade antissocial e ao Transtorno borderline e inclui sentenças relacionadas à conduta invasiva, comportamento de risco, descaso social, agressividade e necessidade de chamar atenção.

A correlação negativa observada entre comportamento pró-social e indicadores de ansiedade é supostamente explicada pelo bem-estar adquirido em situações em que o comportamento social apresentado pelo sujeito é reforçado pela comunidade. Em contrapartida, a correlação positiva obtida entre o fator Desafio-oposicionismo e os fatores Ansiedade e Depressão relaciona-se, provavelmente, às situações de exclusão social que o oposicionismo implica.

Um dado interessante foi a correlação obtida entre o fator Comportamento pró-social" e o Enfrentamento/ 
autoafirmação com risco do Inventário de Habilidades Sociais. Ambos os fatores são compostos por sentenças que representam atenção aos direitos alheios e outros comportamentos que avaliam assertividade social. Além disso, os comportamentos descritos em ambos os fatores parecem depender mais de um autorreforçamento do que de um reforçamento externo.

As correlações positivas obtidas entre a subescala Desafio-oposicionismo e os fatores Conversação e desenvoltura social e Autoexposição a desconhecidos e situações novas do IHS corroboram a ideia de que o terceiro fator da Escala de Conduta Social envolve indicadores de iniciativa social (busca de novidades, determinação, empenho na busca de objetivos planificados, espontaneidade, senso de conquista e ousadia).
Nesse contexto, conclui-se que, a partir da análise da consistência interna e precisão da Escala de Conduta Social e das evidências de validade com base nas correlações com instrumentos padrão ouro para avaliação do construto em questão, a Escala de Conduta Social para Adolescentes é adequada e apresenta evidências de validade para o fim ao qual se propõe. Ainda, apresenta-se como um instrumento útil para a prática clínica e pesquisa, possibilitando, especialmente, o direcionamento de diretrizes clínicas dos adolescentes avaliados. Como limitações do presente estudo, inclui-se a utilização de uma amostra, apesar de abrangente, não representativa de todo o Brasil. Sugere-se, para estudos futuros, que a escala em questão seja aplicada e validada para outros contextos, como o de indivíduos institucionalizados.

\section{Referências}

Almeida, R. M.M., Trentini, L. B., Klein, L. A., Macuglia, G. L., Hammer C., Tesmmer, M. (2014). Uso de álcool, drogas, níveis de impulsividade e agressividade em adolescentes do Rio Grande do Sul. Psico, 45(1), 65-72.

American Psychiatric Association [APA] (2002). Manual diagnóstico e estatístico de transtornos mentais (DSM-IV-TR). Porto Alegre: ArtMed.

Benchaya, M. C., Bisch, N. K., Moreira, T. C., Ferigolo, M., \& Barros, H. M. T. (2011). Non-authoritative parents and impact on drug use: The perception of adolescent children. Jornal de Pediatria, 87(3), 238-244. doi: 10.2223/JPED.2089

Borsa, J. C., Souza, D. S., \& Bandeira, D. R. (2011). Prevalência dos problemas de comportamento em uma amostra de crianças do Rio Grande do Sul. Psicologia Teoria e Prática, 13(2), 15-29.

Bueno, A. C. W., Santos, B. C., \& Moura, C. B. (2010). Obediência infantil: conceituação, medidas comportamentais e resultados de pesquisas. Psicologia: Teoria e Pesquisa, 26(2), 203-216. doi: 10.1590/S0102-37722010000200002

Carneiro, R. S., Falcone, E., Clark, C., Del Prette, Z., \& Del Prette, A. (2007). Qualidade de vida, apoio social e depressão em idosos: relação com habilidades sociais. Psicologia: Reflexão e Crítica, 20(2), 229-237.

Costa, D. L., \& Cavalvante, L. I. (2012). Comportamento de cuidado entre crianças institucionalizadas: observações nos pátios do abrigo e da escola. Gerais, Revista Interinstitucional de Psicologia, 5(1), 50-68.

de Deus, A. S. (2013). Aspectos jurídicos e sociais da criminalidade juvenil: uma análise de estatísticas. Revista da Faculdade Mineira de Direito, 16(32). doi: 10.5752/p.2318-7999.2013v16n32p142

Del Prette, Z., \& Del Prette, A. (2003). Inventário de habilidades sociais (IHA-Del-Prette): manual de aplicação, apuração e interpretação (2a edição revisada e ampliada). São Paulo: Casa do Psicólogo.

Del Prette, Z. A. P., \& Del Prette, A. (2008). Psicologia das habilidades sociais na infância: teoria e prática. Petrópolis: Vozes, $3^{\text {a }}$ edição.

Feitosa, H. N., Ricou, M., Rego, S., \& Nunes, R. (2011). A saúde mental das crianças e dos adolescentes: considerações epidemiológicas, assistenciais e bioéticas. Revista de Bioética, 19(1), 259-275.

Henriques, B. M. (2014). Qualidade da vinculação e comportamento antissocial na infância. International Journal of Developmental and Educational Psychology. INFAD Revista de Psicología, 1, 35-44.

Hutz, C., \& Nunes, C. (2001). Manual da escala de ajustamento emocional/neuroticismo - EFN. São Paulo: Casa do Psicólogo.

International Test Commission [ITC] (2000). Guidelines on test use. Recuperado de https://www.intestcom.org/files/guideline_test_use.pdf

Koch, L., \& Gross, A. (2005). Características clínicas e tratamento do transtorno da conduta. Em V. Caballo \& Miguel Simon (Eds.). Manual de psicologia clínica e do adolescente (pp. 23-38). São Paulo: Santos.

Krintensen, C., Lima, J., Ferlin, M., Flores, R., \& Hackmann, P. (2003). Fatores etiológicos da agressão física: uma revisão teórica. Estudos de Psicologia, 8, 175-184. doi: 10.1590/S1413-294X2003000100020

Laird, R. D., Pettit, G. S., Dodge, K. A., \& Bates, J. E. (2005). Peer relationship antecedents of delinquent behavior in late adolescence: Is there evidence of demographic group differences in developmental processes? Development and Psychopathology, 17, 1-18.

Macedo, A., Paim, J., Silva, L. \& Costa, M. C. (2001). Violência e desigualdade social: mortalidade por homicídios e condições de vida em Salvador, Brasil. Revista de Saúde Pública, 35, 515-522. doi: 10.1590/S0034-89102001000600004

Marques, J. A. M. (2014). Violência e vitimização na infância e adolescência - a inclusão da escola no reconhecimento e prevenção. Ciência e Saúde Coletiva, 19(3), 987-988. doi: 10.1590/1413-81232014193.18372013

Martins, A. F., Silva, K. S., Filho, J. G. B., Ribeiro, M. A., \& Queiroz, A. C. M. (2013). Violence with children and adolescents: Profiles of victims, of aggression and of perpetrators. Revista de Enfermagem UFPI, 2(4), 50-7.

Nardi, F. L., Dell'Aglio, D. D. (2012). Adolescentes em conflito com a Lei: percepções sobre a família. Psicologia: Teoria e Pesquisa, 28(2), 181-191.

Netto, L., Rodrigues, R. N., Costa, M. A., Santos, J., \& Tatagiba, G. A. (2012). Experiências e especificidades da violência escolar na percepção de funcionários de uma escola pública. Revista de Enfermagem UFSM, 2(3), 591-600.

Pacheco, J. T. B., \& Hutz, C. S. (2009). variáveis familiares preditoras do comportamento antissocial em adolescentes autores de atos infracionais. Psicologia: Teoria e Pesquisa, 25(2), 213-219. doi: 10.1590/S0102-37722009000200009 
Pacheco, J., Alvarenga, P., Reppold, C.T., Piccinini, C.A., \& Hutz, C.S. (2005). Estabilidade do comportamento antissocial na transição da infância para a adolescência: uma perspectiva desenvolvimentista. Psicologia: Reflexão e Crítica, 18(1), 55-61. doi: 10.1590/S010279722005000100008

Pasquali, L. (1999). Instrumentos psicológicos: manual prático de elaboração. Brasília: LabPAM/IBAP.

Paula, A. S., Kodato, S., \& Dias, F. X. (2013). Representações sociais da violência em professores da escola pública. Estudos interdisciplinares em psicologia, 4(2), 240-257.

Pedrini, J. R., \& Frizzo, G. B. (2010). Evaluation of child behavior problems indicators reported by parents and teachers. Aletheia, 33 , 69-83.

Rocha, M. M. (2012). Evidências de validade do inventário para adolescentes (YSR/2001) para a população brasileira (Tese de doutorado não publicada). Universidade de São Paulo, São Paulo.

Silva, L. R. F. (2011). Transtorno da conduta: uma oportunidade para a prevenção em saúde mental? Interface, 15(36), 65-173. doi: 10.1590/ S1414-32832011000100013.

Silva, R. A., Cardoso, T. A., Jansen, K., Souza, L. D. M., Godoy, R. V., Cruzeiro, A. L. S., ... Pinheiro, R. T. (2012). Bullying and associated factors in adolescents aged 11 to 15 years. Trends Psychiatry Psychotherapy, 34(1), $19-24$.

Souza, C. C., \& Resende, A. C. (2012). Transtornos psicológicos em adolescentes socioeducandos. Avaliação Psicológica, 11(1), 95-109.

Teichner, G., \& Golden, C. J. (2000). The relationship of neuropsychological impairment to conduct disorder in adolescence: A conceptual review. Aggression and Violent Behavior, 5, 509-528. doi: 10.1016/S1359-1789(98)00035-4

Zanon, C., \& Hutz, C. S. (2009). Propriedades psicométricas da Escala Fatorial de Neuroticismo e do questionário de ruminação e reflexão. Avaliação Psicológica, 8(2), 279-281.

\section{Sobre os autores}

Caroline Tozzi Reppold é psicóloga, com pós-doutorado em Psicologia pela UFRGS e em avaliação psicológica pela Universidade São Francisco. Professora associada I da Universidade Federal de Ciências da Saúde de Porto Alegre. Docente permanente dos PPG Ciências da Saúde e PPG Ciências de Reabilitação/UFCSPA. Coordenadora do Laboratório de Pesquisa em Avaliação Psicológica da UFCSPA. Bolsista Produtividade do CNPq.

Léia Gonçalves Gurgel é fonoaudióloga, mestre e doutoranda do Programa de Pós-graduação em Ciências da Saúde da Universidade Federal de Ciências da Saúde de Porto Alegre. Bolsista de doutorado da CAPES.

Claudio Simon Hutz é psicólogo, doutor pela University of Iowa (USA) e Pós-doutor pela Arizona State University (USA). Professor titular da Universidade Federal do Rio Grande do Sul. Docente permanente do PPG Psicologia/UFRGS. Coordenador do Laboratório de Mensuração da UFRGS. Presidente da Associação Brasileira de Psicologia Positiva (ABP+). Bolsista Produtividade do CNPq. 